\title{
Force on a sphere via the generalized reciprocal theorem
}

\author{
A. M. Leshansky and J. F. Brady a) \\ Division of Chemistry \& Chemical Engineering, California Institute of Technology, \\ Pasadena, California 91125
}

(Received 28 October 2003; accepted 12 December 2003; published online 4 February 2004)

\begin{abstract}
An approach based on the generalized reciprocal theorem is presented to derive the well-known result for the drag force exerted on a rigid sphere translating in a viscous fluid in an arbitrary manner. The use of generalized reciprocal theorem allows one to bypass the calculation of stress distribution over the particle surface in order to compute the force. (c) 2004 American Institute of Physics. [DOI: 10.1063/1.1645491]
\end{abstract}

Suppose that a rigid spherical particle with radius $R$ undergoes translatory oscillations in a viscous fluid. When the Reynolds number of the oscillatory motion is small, Re $=\omega R \ell / \nu \ll 1$, the convective terms, $(\mathbf{v} \cdot \nabla) \mathbf{v}$, in the NavierStokes equations can be neglected. Here $\omega$ and $\ell$ are the frequency and the amplitude of the oscillations, respectively, and $\nu$ is a kinematic viscosity of the fluid. We also take the oscillations to have a small amplitude, $\ell / R \ll 1$. Under these conditions, the fluid vorticity, $\boldsymbol{\zeta}=\boldsymbol{c u r l} \mathbf{v}$, satisfies the heat equation

$$
\partial \zeta / \partial t=\nu \Delta \zeta .
$$

Following Landau and Lifshitz ${ }^{1}$ we write the velocity of the sphere in the form $\mathbf{u}=\mathbf{u}_{0} e^{-i \omega t}$, where $\mathbf{u}_{0}$ is a constant vector. From the linearity of the problem the velocity of the fluid around the sphere has the form

$$
\mathbf{v}=\mathrm{e}^{-\mathrm{i} \omega t} \text { curl curl } g \mathbf{u}_{0},
$$

where $g$ is a function of a radial coordinate $r$ only, while the instantaneous origin is taken at the center of the sphere. Substituting (2) into (1) and choosing the physically realizable solution (which decays exponentially with $r$ ) one obtains

$$
g^{\prime}(r)=\left(a \mathrm{e}^{\mathrm{i} k r}(r-1 / \mathrm{i} k)+b\right) / r^{2},
$$

where $k=(1+\mathrm{i}) / \delta$ and $\delta=\sqrt{2 \nu / \omega}$ is the penetration depth. The constants $a$ and $b$ are found by applying the no-slip boundary condition $\mathbf{v}=\mathbf{u}$ at $r=R$ (see Problem 5 on p. 89 in Landau and Lifshitz ${ }^{1}$ )

$$
a=-\frac{3 R}{2 \mathrm{i} k} \mathrm{e}^{-\mathrm{i} k R}, \quad b=-\frac{1}{2} R^{3}\left(1-\frac{3}{\mathrm{i} k R}-\frac{3}{k^{2} R^{2}}\right) .
$$

The reciprocal theorem for low Reynolds number flows allows one to bypass the detailed solution of the problem by relating the hydrodynamic force on a particle to an integral over the particle surface of the velocity field in which the particle is immersed. A generalization of this theorem for arbitrary Reynolds numbers is possible, with the effect of inertia now appearing as a volume integral. ${ }^{2}$ From Lovalenti

${ }^{a)}$ Electronic mail: jfb@cheme.caltech.edu and $\mathrm{Brady}^{2}$ the force exerted on a particle by the fluid in a coordinate frame instantaneously fixed with the particle's center is given by

$$
\mathbf{F}=\mathbf{F}^{S}-\rho \int_{V_{f}} \mathcal{M} \cdot \mathbf{f} \mathrm{d} V(\mathbf{x}) .
$$

Here $\mathbf{F}^{S}$ is the pseudo-steady Stokes drag on the particle, $\mathcal{M}$ is a second-rank Stokes mobility tensor, $\mathbf{f}(\mathbf{x})$ represents the inertia terms in the equation of motion and for the smallamplitude transient motion considered here $\mathbf{f}=\partial \mathbf{v} / \partial t$. The integral in (5) is over the entire fluid volume $V_{f}:=\{\mathbf{x}$ $\left.\in \mathbb{R}^{3}:|\mathbf{x}|>R\right\}$. For a spherical particle, $\mathbf{F}^{S}=-6 \pi R \eta \mathbf{u}(t)$ and $\mathcal{M}$ is given by

$$
\mathcal{M}_{i j}(\mathbf{x})=\frac{3 R}{4}\left(\frac{\delta_{i j}}{r}+\frac{x_{i} x_{j}}{r^{3}}\right)+\frac{R^{3}}{4}\left(\frac{\delta_{i j}}{r^{3}}-\frac{3 x_{i} x_{j}}{r^{5}}\right) .
$$

From (2) and (3) the velocity field around the particle is

$$
v_{i}=\left\{G_{1}(r)\left(u_{i}-\frac{x_{i} x_{m} u_{m}}{r^{2}}\right)-G_{2}(r) u_{i}\right\} \mathrm{e}^{-\mathrm{i} \omega t},
$$

where

$$
G_{1}(r)=\frac{g^{\prime}(r)}{r}-g^{\prime \prime}(r), \quad G_{2}(r)=\frac{2 g^{\prime}(r)}{r},
$$

and $g^{\prime}(r)$ is given in (3). Differentiating (7) with respect to time and substituting into the volume integral in (5) we find that the unsteady inertia contribution to the force reads

$$
\int_{r>R} \mathcal{M}_{i k} \frac{\partial v_{k}}{\partial t} \mathrm{~d} V(\mathbf{x})=-\mathrm{i} \omega \mathrm{e}^{-\mathrm{i} \omega t} u_{i} \mathcal{I},
$$

and the complex constant $\mathcal{I}$ is given by

$$
\begin{aligned}
\mathcal{I} & =4 \pi \int_{r=R}^{\infty}\left\{G_{1}(r) \frac{3 r^{2} R+R^{3}}{6 r^{3}}-G_{2}(r) \frac{R}{r}\right\} r^{2} \mathrm{~d} r \\
& =(3+3 \mathrm{i}) \pi \delta R^{2}+\frac{2 \pi R^{3}}{3} .
\end{aligned}
$$

It is readily seen from (9) and (10) that the unsteady inertia has two contributions to the drag force: the imaginary part of $\mathcal{I}$ results in a contribution proportional to $\mathbf{u}$ and the real part of $\mathcal{I}$ produces a term proportional to $\dot{\mathbf{u}}$ giving 


$$
\begin{aligned}
\rho \int_{V_{f}} \mathcal{M} \cdot \mathbf{f} d V(\mathbf{x}) & =\rho \frac{2 \nu}{\delta^{2}} \mathbf{u} \operatorname{Im}\{\mathcal{I}\}+\rho \dot{\mathbf{u}} \operatorname{Re}\{\mathcal{I}\} \\
& =\frac{6 \pi \eta R^{2}}{\delta} \mathbf{u}+3 \pi \delta \rho R^{2}\left(1+\frac{2 R}{9 \delta}\right) \dot{\mathbf{u}}
\end{aligned}
$$

where we used the substitution $\omega=2 \nu / \delta^{2}$. Finally, combining this result with (5) the net force on the sphere reads

$$
\mathbf{F}=-6 \pi \eta R\left(1+\frac{R}{\delta}\right) \mathbf{u}-3 \pi \delta \rho R^{2}\left(1+\frac{2 R}{9 \delta}\right) \dot{\mathbf{u}}
$$

which is identical to the expression (3) on p. 89 in Landau and Lifshitz, ${ }^{1}$ where the total force exerted on the sphere was calculated directly by integration of the local force density, $\boldsymbol{\sigma} \cdot \mathbf{n}$, over the surface of the sphere. Thus, the use of the generalized reciprocal theorem bypasses the need to calculate the fluid stress $\boldsymbol{\sigma}$.

The force exerted on a sphere translating in an arbitrary manner (still with $\mathrm{Re} \ll 1$ ) with its velocity given as a function of time, $\mathbf{u}(t)$, can be determined in the same fashion; we may represent $\mathbf{u}(t)$ as a Fourier integral:

$$
\mathbf{u}(t)=\frac{1}{2 \pi} \int_{-\infty}^{\infty} \hat{\mathbf{u}}_{\omega} \mathrm{e}^{-\mathrm{i} \omega t} \mathrm{~d} \omega, \quad \hat{\mathbf{u}}_{\omega}=\int_{-\infty}^{\infty} \mathbf{u}(\tau) \mathrm{e}^{\mathrm{i} \omega \tau} \mathrm{d} \tau .
$$

Since the equations are linear, operations of time differentiation and spatial integration commute, which allows the force on a sphere to be expressed as the integral over the whole spectrum of forces corresponding to separate Fourier components of the velocities, $\hat{\mathbf{u}}_{\omega} \mathrm{e}^{-i \omega t}$. Thus, we may re-write (5) as

$$
\mathbf{F}=-6 \pi R \eta \mathbf{u}(t)-(2 \pi)^{-1} \rho \int_{-\infty}^{\infty} \int_{V_{f}} \mathcal{M} \cdot \hat{\mathbf{f}} \mathrm{d} V(\mathbf{x}) \mathrm{d} \omega,
$$

where $\hat{\mathbf{f}}$ is now calculated from (7) with $u_{i}$ being replaced by $\hat{u}_{i}$. Taking into account (9) and (10) the volume integral in (13) becomes

$$
\begin{aligned}
& (2 \pi)^{-1} \int_{-\infty}^{\infty} \int_{r>1} \mathcal{M} \cdot \hat{\mathbf{f}} \mathrm{d} V(\mathbf{x}) \mathrm{d} \omega \\
& \quad=(2 \pi)^{-1} \int_{-\infty}^{\infty} \mathrm{i} \omega \pi R^{3} \mathrm{e}^{-\mathrm{i} \omega t} \hat{\mathbf{u}}_{\omega}\left(\frac{2}{3}+\frac{3 \sqrt{2 \nu}}{R} \frac{1+\mathrm{i}}{\sqrt{\omega}}\right) \mathrm{d} \omega .
\end{aligned}
$$

The first term under the integral sign can be immediately evaluated by noting that $(\widehat{\mathrm{du} / \mathrm{d} t})_{\omega}=-\mathrm{i} \omega \hat{\mathbf{u}}_{\omega}$, and integrating over $\omega / 2 \pi$ results in $-2 \pi R^{3} \dot{\mathbf{u}}(t) / 3$. The second term under the integral sign can be integrated in exactly the same way as in Landau and Lifshitz, ${ }^{1}$ p. 90, Problem 7, and after some algebra gives

$$
\operatorname{Re}\left\{\frac{(1+\mathrm{i})}{2 \pi} \int_{-\infty}^{\infty} \frac{\widehat{(\dot{\mathbf{u}})}{ }_{\omega} \mathrm{e}^{-\mathrm{i} \omega t}}{\sqrt{\omega}} \mathrm{d} \omega\right\}=\sqrt{\frac{2}{\pi}} \int_{-\infty}^{t} \frac{\dot{\mathbf{u}}(\tau)}{\sqrt{t-\tau}} \mathrm{d} \tau .
$$

Substituting into (13) we finally obtain the classical formula for the drag

$$
\begin{aligned}
\mathbf{F}= & -6 \pi R \eta \mathbf{u}(t)-\frac{2}{3} \pi \rho R^{3} \dot{\mathbf{u}}(t) \\
& -6 \pi \rho R^{2} \sqrt{\frac{2}{\pi}} \int_{-\infty}^{t} \frac{\dot{\mathbf{u}}(\tau)}{\sqrt{t-\tau}} \mathrm{d} \tau,
\end{aligned}
$$

where the second term is the so-called "added mass" contribution and the last term is the Basset force.

The presented approach provides an elegant short-cut in deriving the unsteady force exerted on a sphere undergoing an arbitrary time-dependent motion at low Reynolds numbers since it does not require the calculation of the viscous stress and pressure distribution on the sphere surface. It may be useful in other context such as the motion of nonspherical particles or particle-particle interactions in a suspension. Finally, the generalized reciprocal theorem (5) with $\mathbf{f}$ given by the complete fluid acceleration is valid for an arbitrary frequency, provided that the effect of the convective inertia is negligible.

${ }^{1}$ L. D. Landau and E. M. Lifshitz, Fluid Mechanics, 2nd ed. (Pergamon, New York, 1987).

${ }^{2}$ P. M. Lovalenti and J. F. Brady, "The hydrodynamic force on a rigid particle undergoing arbitrary time-dependent motion at small Reynolds number,” J. Fluid Mech. 256, 561 (1993). 